\title{
自由基极性反转策略实现的烯烃烷基化反应
}

\author{
韦榕标＼cjkstart鲍红丽* \\ (中国科学院福建物质结构研究所 福州 350002)
}

\section{Polarity Umpolung Strategy for the Radical Alkylation of Alkenes}

\author{
Wei, Rongbiao Bao, Hongli*
}

(Fujian Institute of Research on the Structure of Matter, Chinese Academy of Sciences, Fuzhou 350002)

烯烃是石油产业的副产物, 廉价易得, 在工业生产 及科学研究中广泛应用. 近年来, 由于烯烃的双官能团 化可以同时构建两个新的化学键受到越来越多的关注, 其已成为合成化学中重要的策略来构建结构复杂多样、 官能团丰富的有机化合物. 尽管在该领域有很多突破, 烯烃化合物的烷基化双官能团化反应却仍然存在一些 问题需要解决. 比如, 使用过渡金属催化剂时, 存在 $\beta$ - $\mathrm{H}$ 的烷基与金属结合后生成的 $\mathrm{M}-\mathrm{C}\left(\mathrm{sp}^{3}\right)$ 中间体易发 生 $\beta-\mathrm{H}$ 消除反应, 从而得不到烯烃双官能团化反应产 物 ${ }^{[1]}$. 近些年, 随着自由基化学的长足发展, 烯烃化合 物的自由基型烷基化反应为上述问题提供了一种快捷 而有效的方法.

总体上，烯烃的自由基烷基化反应主要有两种模 式. 一种是极性匹配模式(Polarity-Matched Mode): 即亲 核的烷基自由基对缺电子的烯烃进行自由基加成(Giese 反应) ${ }^{[2]}$. 另一种模式为 $p-\pi$ 共轭辅助模式( $\mathrm{p}-\pi$ Conjugation Assisted Mode $)^{[3 \sim 6]}$. 烷基自由基进攻烯烃, 再通过 $\mathrm{p}-\pi$ 共轭作用得到热力学上稳定的自由基中间体, 比如 常见的苄基自由基中间体. 因此, 上述两种模式均只对 特异性的烯烃化合物有较好的效果, 而对非活化的非特 异性的烯烃则效果不佳. 目前对此类非活化的非特异性 的普通烯烃的烷基化反应仍具有挑战.

苏州大学化学学院朱晨课题组近些年利用自由基 迁移策略开发了一系列高效的烯烃自由基双官能团化 反应 ${ }^{[7]}$, 并创造性地发展了一种全新的对接一迁移模式 (docking-migration strategy $)^{[8]}$. 最近, 在前期工作的基础 上, 朱晨课题组 ${ }^{[9]}$ 实现了上述具有挑战的普通烯烃的自 由基烷基化反应. 作者巧妙地将烷基基团连接在砜基 上, 利用对接-迁移模式和自由基物种的极性反转策略, 实现了光引发的普通烯烃的自由基烷基化双官能团化
反应(Scheme 1).

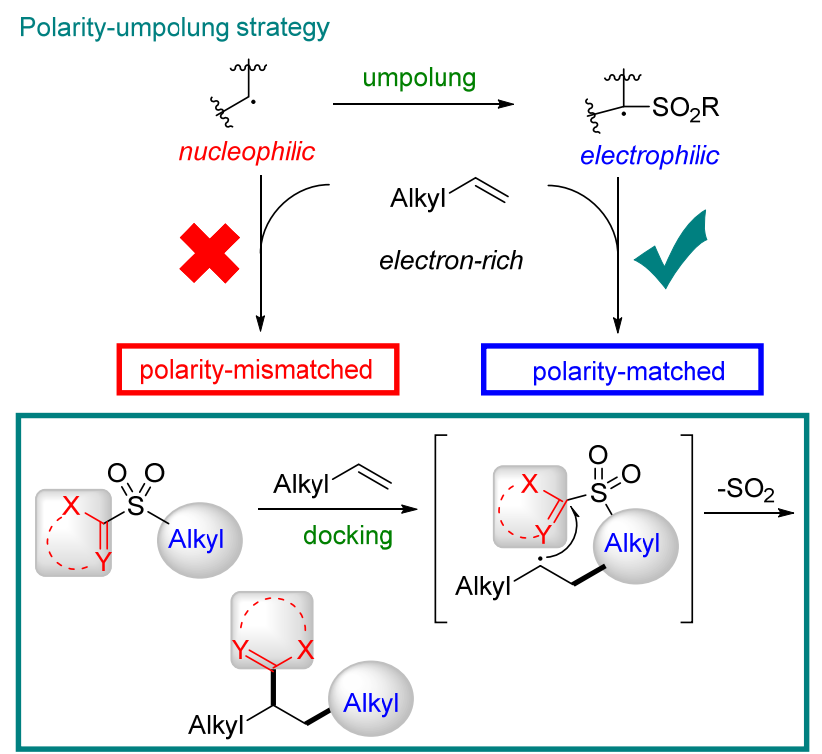

图 1 自由基介导的烯烃烷基化

Scheme 1 Radical-mediated alkylation of alkenes

该反应不但适用于普通烯烃的自由基烷基杂芳烃 化反应，而且也能拓展至自由基烷基肟基化反应. 研究 发现, 硫醇的作用至关重要, 因为它既可以为反应提供 氢源, 又可以作为反应的内在还原剂. 无机碱的添加可 中和原位生成的氢溴酸, 从而显著提高产物收率. 在烯 烃的自由基烷基肜基化反应中, 路易斯酸(比如 $\mathrm{LiCl}$ )可 以与双官能团试剂相互作用, 从而改善此试剂的反应活 性. 值得注意的是，在日光照射下烯烃的自由基烷基杂 芳烃化反应也能进行，很好地证明其极佳的反应效率和 生态相容性.

对烯烃化合物的自由基烷基杂芳基化和烷基肜基

* Corresponding author. E-mail: hlbao@fjirsm.ac.cn. Published online May 4, 2020. 
化反应的底物范围的考察结果表明, 该方法具有广泛的 底物范围和出色的官能团耐受性(Scheme 2). 而且, 除 了普通烯烃, 活化烯烃也能实现此自由基烷基化反应. 研究表明, 由于末端烯烃具有较高的电子密度和较小的 空间位阻, 在烷基自由基加成步骤中, 末端烯烃相比于 $\alpha, \beta$-不饱和烯烃和内烯具有更好的化学选择性. 其次, 在体系中加入 $\mathrm{D}_{2} \mathrm{O}$ 时, 反应能以优良的氞代率得到忥代 烷基化产物. 复杂的天然产物或药物衍生物在该体系下 也能顺利实现自由基烷基化反应. 此外, $\mathrm{SO}_{2}$ 分子脱除 后生成的新烷基自由基还可以再次被其他自由基捕集 剂拦截, 从而实现多官能团加合物的形成.

通过反应物氧化还原电势的测定并结合苂光淬灭 实验, 推测反应中激发态的铱催化剂 $\mathrm{Ir}^{\mathrm{III} *}\left(E_{1 / 2}{ }^{\mathrm{III} / \mathrm{IV}}=-\right.$ $1.73 \mathrm{~V}$ vs. SCE) 是被砜基化合物氧化淬灭的. 光开/关实 验表明反应的主要途径可能是自由基链反应. 拉曼试验 观察到了 $\mathrm{S}-\mathrm{Br}$ 和 $\mathrm{S}-\mathrm{S}$ 键信号, 进一步证明反应可能 为链式反应. 此外, 计算表明双官能团化试剂中砜基的
存在对于溴原子的篗取至关重要, 这可能是因为砜基能 够稳定溴篗取后得到的自由基中间体.

根据实验及计算结果, 作者提出了一种可能的反应 机理(Scheme 3). 光催化剂吸收光子被激发, 进而还原 化合物 1 或 9 的 $\mathrm{C}-\mathrm{Br}$ 键，生成的烷基自由基 $\mathbf{A}$ 随后引 发自由基串联反应. 首先, 物种 $\mathbf{A}$ 对烯烃亲电加成生成 自由基 $\mathbf{B}$ 物种. 自由基 $\mathbf{B}$ 随后被杂芳基或肜基捕获, 发 生分子内迁移得到中间体 $\mathbf{C}$. 中间体 $\mathbf{C}$ 释放一分子 $\mathrm{SO}_{2}$ 后得到中间体 $\mathbf{D}$. 接着中间体 $\mathbf{D}$ 与硫醇反应得到产物. 同时，新生成的颈基自由基与双官能试剂 1 或 9 反应直 接生成自由基物种 $\mathbf{A}$, 使得自由基链反应得以继续进 行.

总结, 朱晨课题组利用自由基极性反转策略, 实现 了具有挑战的普通烯烃的烷基化官能团化反应. 反应条 件温和，官能团耐受性好，底物广泛，机理研究清晰， 具有启发性. 此方法为扩展脂肪族骨架提供了一种有效 的方法.
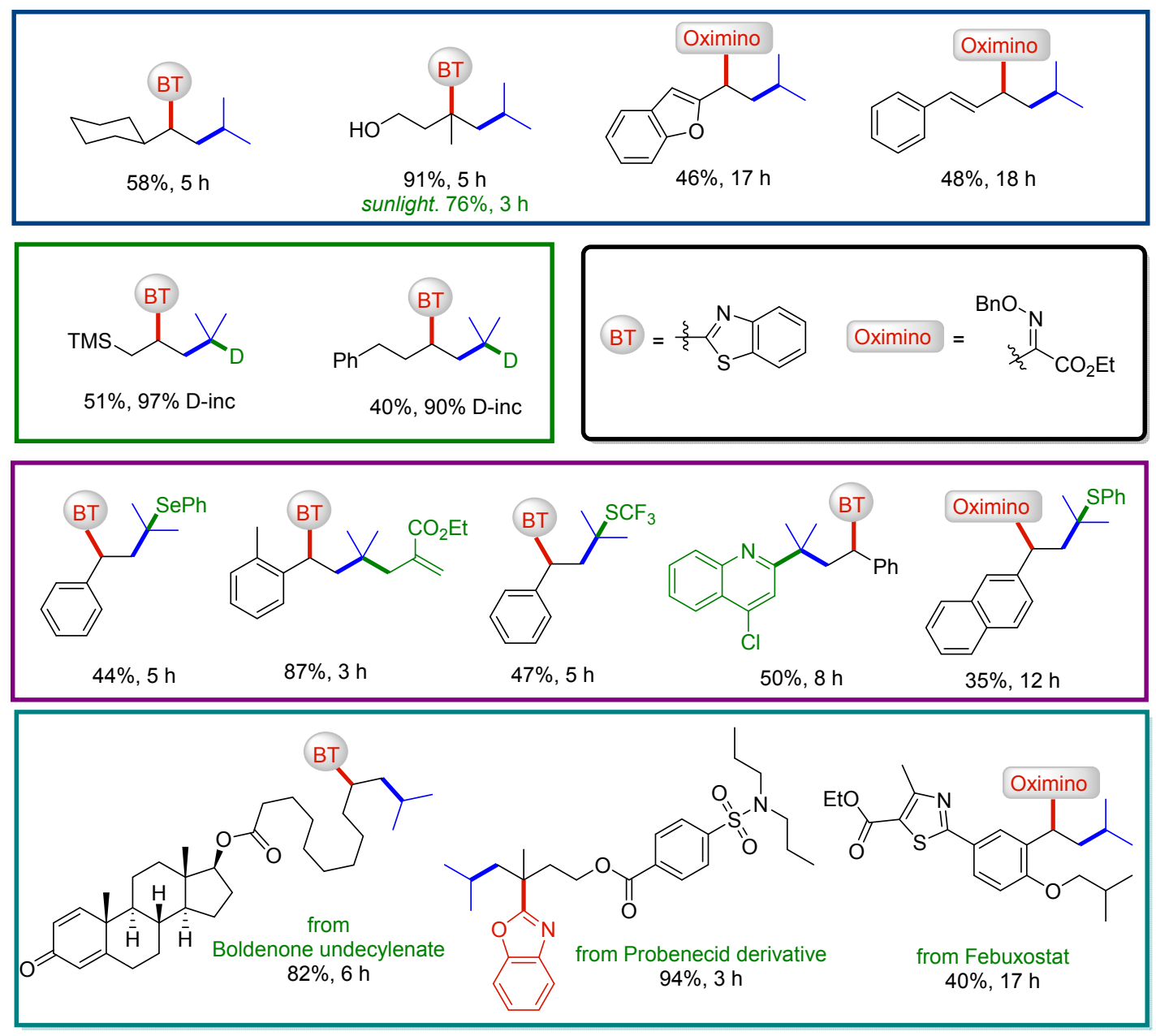

图式 2 部分底物拓展

Scheme 2 Part of the substrate scope 


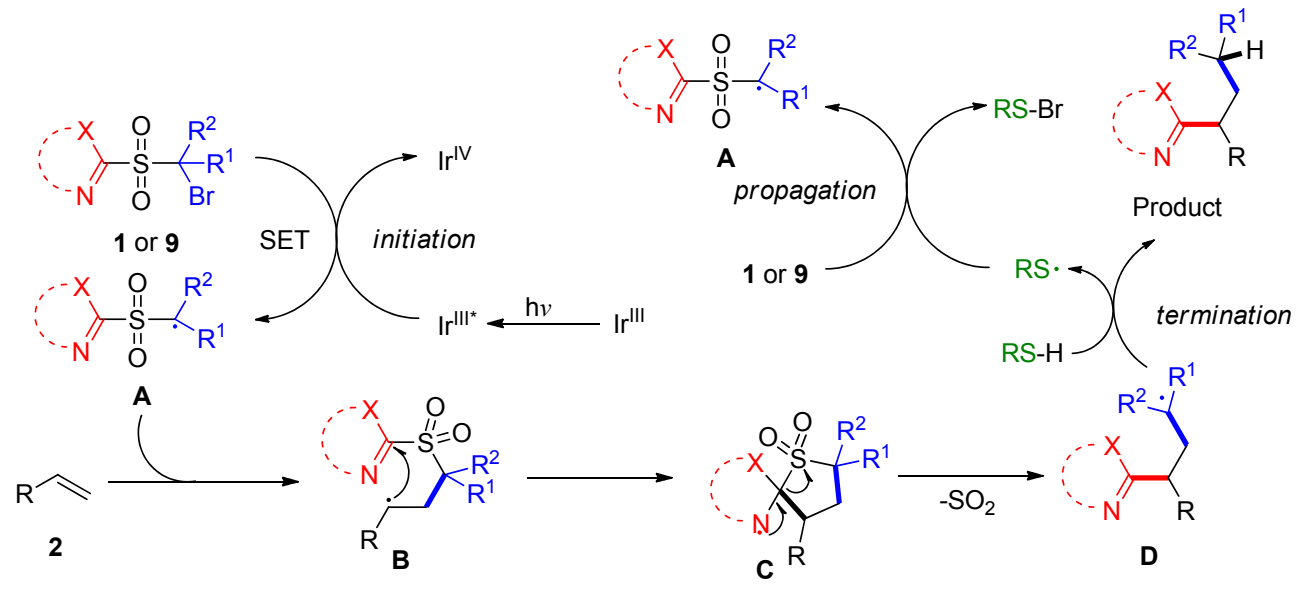

图式 3 可能的催化机理

Scheme 3 Possible reaction mechanism

\section{References}

[1] Luh, T.-Y.; Leung, M.-K.; Wong, K.-T. Chem. Rev. 2000, 100, 3187.

[2] Giese, B.; González-Gómez, J. A.; Witzel, T. Angew. Chem., Int. Ed. 1984, 23, 69.

[3] Jian, W.; Ge, L.; Jiao, Y.; Qian, B.; Bao, H. Angew. Chem., Int. Ed. 2017, 56, 3650.

[4] Qian, B.; Chen, S.; Wang, T.; Zhang, X.; Bao, H. J. Am. Chem. Soc.
2017, 139, 13076.

[5] Bunescu, A.; Ha, T. M.; Wang, Q.; Zhu, J. Angew. Chem., Int. Ed. 2017, 56, 10555 .

[6] Bao, X.; Yokoe, T.; Ha, T. M.; Wang, Q.; Zhu, J. Nat. Commun. 2018, 9, 3725.

[7] Wu, X.; Zhu, C. Chem. Commun. 2019, 55, 9747.

[8] Yu, J.; Wu, Z.; Zhu, C. Angew. Chem., Int. Ed. 2018, 57, 17156.

[9] Liu, J.; Wu, S.; Yu, J.; Lu, C.; Wu, Z.; Wu, X.; Xue, X. S.; Zhu, C. Angew. Chem., Int. Ed. 2020, 59, 8195.

(Cheng, F.) 